\title{
Detection of Escherichia coli Bacteria by Impact Electrochemistry
}

Rosa A.S. Couto ${ }^{a, \dagger}$, Lifu Chen ${ }^{b, \dagger}$, Sabine Kuss ${ }^{b}$, and Richard G. Compton ${ }^{b *}$

${ }^{a}$ LAQV/REQUIMTE, Department of Chemical Sciences, Faculty of Pharmacy, University of Porto, Porto, Portugal.

${ }^{b}$ Department of Chemistry, Physical and Theoretical Chemistry Laboratory, University of Oxford, South Parks Road, Oxford, OX1 3QZ, UK.

\section{Corresponding Author}

*Correspondence to: richard.compton@chem.ox.ac.uk

Telephone number: +44(0) 1865275957

${ }^{\dagger}$ : These authors contributed equally to the paper.

To be submitted to:

Analyst 


\section{ABSTRACT}

We report the redox mediated detection of Escherichia coli bacteria at carbon microelectrodes, using the impact electrochemistry technique. By employing N,N,N',N'tetramethyl-para-phenylene-diamine (TMPD) as redox mediator a concentration dependency for bacteria impacts was observed, whereby its impact frequency is shown to be in good agreement with theoretically predicted values. 
The design and development of biosensors for the detection of bacteria is gaining increasing attention, as bacterial infections represent one of the leading causes of mortality worldwide. ${ }^{1,2}$ In recent years, chemical and electrochemical sensors have become particularly attractive, mainly due to their characteristics in high sensitivity, low cost and rapid output. ${ }^{3-6} \mathrm{~A}$ number of promising approaches have been proposed for the detection of bacterial by-products, metabolites, as well as enzymes. ${ }^{3,4}$

The detection of Escherichia coli (E. coli) is crucial, as contaminations with this pathogen play a significant role in the food industry, the environment and the health sector. Standard guidelines for sample limits have been introduced. ${ }^{7,} 8$ As an example, the United Kingdom currently defines a zero standard for all E. coli organisms in drinking water ${ }^{9}$, whereas the threshold for a clinical significance in regards to urinary tract infections related to $E$. coli has been reported to be at a level of $10^{5}$ culture forming units (CFU) $\mathrm{mL}^{-1} \cdot{ }^{10}$ Hence, developing detection methods that are able to recognize infections at higher concentrations is important and needed. As current analysis procedures take at least $18 \mathrm{~h}$ from sample collection to result ${ }^{11}$, an accurate, sensitive, rapid and cost efficient alternative for the detection of $E$. coli is needed.

Impact electrochemistry is a powerful technique for the detection of single entities $^{12,} 13$ and can be illustrated by the detection and characterization of silver nanoparticles in solution. ${ }^{14}$ Its principle is based on the Faradaic charge transfer, following the collision of redox active entities on the nano- or micrometer scale with an electrode. Governed by diffusional Brownian motion, single particles collide with the electrode, which is held at an oxidizing or reducing potential, exceeding the standard potential of the redox species or material of the particle. Thereby, entity impacts at the electrode result in a short current burst ("spike”). Worth noting is the 
recent application of impact electrochemistry to the qualification and quantification of the locomotion of self-propelled micromotors. ${ }^{15,16}$

The main challenge for the application of impact electrochemistry to biological samples is the interference of the electrochemical signal with biological redox species present in an analyte solution, and their potential absorbance at the electrode surface. To date, no redox active species, interacting directly with bacteria has been reported for the application to impact electrochemistry, but methodologies involving nanoparticle-labelled bacteria and their detection through an electrochemical "off”signal have been proposed. A first approach to detect bacteria using impact electrochemistry was reported by Sepunaru et al. ${ }^{17}$ In this study, E. coli bacteria were labelled with silver nanoparticles, rendering them electroactive upon collision with an electrode. By monitoring the impact frequency depending on bacterial concentrations, it was concluded that this electrochemical approach has the ability to reveal information about the concentration of pathogens in a sample solution. In 2016, the use of nanoparticles could be successfully avoided when Lee at al. reported the labelfree detection of $E$. coli, using the redox couple ferrocyanide/ferricyanide, which is oxidized at carbon fibre microelectrodes. ${ }^{18}$ In this study, target bacteria collide with the electrode blocking the diffusion of the mediator towards the electrode. Although this approach simplifies the overall methodology significantly, the electrochemical detection of bacteria in this example relies on an "off”-signal, which carries a risk for false positive results, for example due to contamination of the analyte solution with any other electrochemically inactive particles of micrometre size.

The detection of ensembles of whole bacterial cells by an electrochemical “on"signal has been demonstrated recently by our group ${ }^{19}$, where the use of N,N,N',N'- 
tetramethyl-para-phenylene-diamine (TMPD) enables the electrochemical recognition of bacterial cytochrome c oxidases without the need of cell lysis. Herein we report the combination of the TMPD-mediated detection of bacteria with the impact electrochemistry methodology. Concentration dependent electrochemical measurements reveal an impact frequency close to the theoretical value, which assumes viable bacteria in solution: For the first time bacteria are detected label-free by an electrochemical “on”-signal using the impact method.

To assure stable diffusion rates, as well as bacterial metabolic conditions, all measurements were conducted inside a thermostated $\left(37^{\circ} \mathrm{C}\right)$ Faraday cage. The three electrode system consisted of a $44 \mu \mathrm{m}$ diameter carbon working electrode, a graphite counter and saturated calomel reference electrode (SCE). The working electrode was polished following an established procedure ${ }^{20}$, and the electrochemical current was recorded using an in-house fabricated low noise potentiostat ${ }^{21}$ and PyFemto software (version 0.6), applying a post-acquisition Bessel filtering of $25 \mathrm{~Hz}$. Following the procedure in literature ${ }^{19}$, E. coli bacteria (courtesy of Prof F.A. Armstrong, Oxford University) were grown in broth culture medium until an optical density between 1.0 and 1.3 was reached, growth medium was removed by centrifugation at $3000 \mathrm{rpm}$ for 15 min and resuspension in PBS. The number of bacteria in solution was determined by optical density $(\mathrm{OD})$ at a wavelength of $600 \mathrm{~nm}\left(\mathrm{OD}_{600}\right.$ of $1.0=8 \times 10^{8}$ cells $\mathrm{mL}^{-}$ $\left.{ }^{1}\right)^{22}$

As TMPD in its reduced form is rapidly oxidised by atmospheric oxygen, the radical cation $\mathrm{TMPD}^{+\bullet}$ was synthesized ${ }^{23}$ in the form of TMPD-tetrafluoroborate (TMPD$\mathrm{BF}_{4}$ ) to provide solution stability and higher accuracy during bioelectrochemical

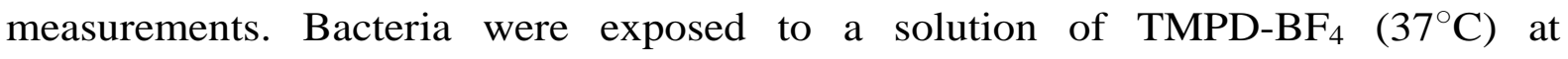


concentrations between 0.7 and $1.0 \mathrm{mM}$. This concentration rage is in accordance with our previous publication ${ }^{19}$, where the detection of dropcasted E. coli bacteria was achieved at macroelectrodes using these TMPD concentrations. The synthesis product TMPD-BF 4 was electrochemically characterized and revealed a ratio of $80 \%$ TMPD$\mathrm{BF}_{4}$ and 20\% TMPD. For all experiments, a 45-second delay time was respected prior to applying a reducing potential of $-0.15 \mathrm{~V}$ at the working electrode. In principle and as shown in scheme 1, bacteria in solution diffuse by Brownian motion towards the microelectrode's reactive surface. By applying a reducing potential at the electrode, $\mathrm{TMPD}^{+\cdot}$ is reduced to TMPD within the diffusion layer. Bacteria, expressing cytochrome c oxidases, are able to oxidize TMPD to $\mathrm{TMPD}^{+\bullet}$, creating a feedback redox cycle when entering the diffusion layer. Consequently, the recorded current increases until all regenerated $\mathrm{TMPD}^{+\bullet}$ in the periphery of the bacterium is consumed by the electrode.

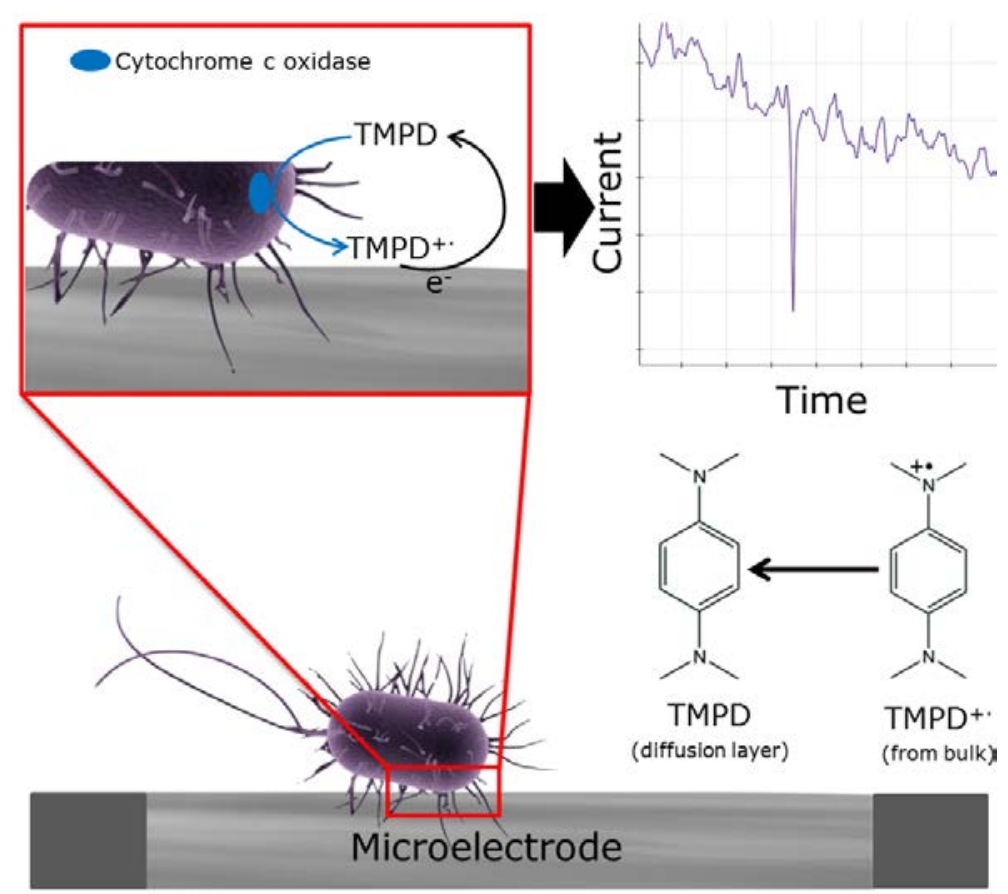

Scheme 1: Schematic representation of E. coli detection by impact electrochemistry. Bacteria are diffusing towards the microelectrode, oxidizing TMPD to TMPD ${ }^{+\bullet}$, which is regenerated at the electrode, resulting in a reductive current spike. 
Results presented in Figure 1 show representative chronoamperometric measurements in the presence (red) and absence (black) of E. coli bacteria. Chronoamperometry was carried out in regular intervals of each 50 seconds. No electrochemical features indicating any bacteria were seen in the control (black curve). In the presence of bacteria in solution, shown here at the example of $1.8 \times 10^{9}$ bacteria $\mathrm{mL}^{-1}$ (red curve), distinctive current spikes are observed. Spikes were counted for all recorded intervals and averaged for each concentration condition. Current spike magnitudes are two to three times the standard deviation above the background current. The shape of the spike, but not the charge, is controlled by the electronics ${ }^{24}$ and appears with an onset and gradual relaxation to the current baseline. This behaviour indicates a reduction of $\mathrm{TMPD}^{+\cdot}$ (in addition to the bulk concentration) provided by the approaching and impacting bacteria, and then back diffusion of E. coli away from the electrode and into the bulk solution.

To test the selectivity of the method against dead bacteria, $1.8 \times 10^{9}$ bacteria $\mathrm{mL}^{-1}$ E. coli were exposed to $1 \%$ Virkon solution for $10 \mathrm{~min}$. This disinfection procedure was reported to result in intracellular protein unfolding and aggregation, leading to cell death. ${ }^{25}$ Virkon was removed from bacteria by centrifugation at $3000 \mathrm{rpm}$ for $15 \mathrm{~min}$ and resuspension of bacteria in PBS. As shown in figure 1 (blue curve), no spikes were recorded under these conditions, indicating no cytochrome c oxidase activity in dead bacteria. 


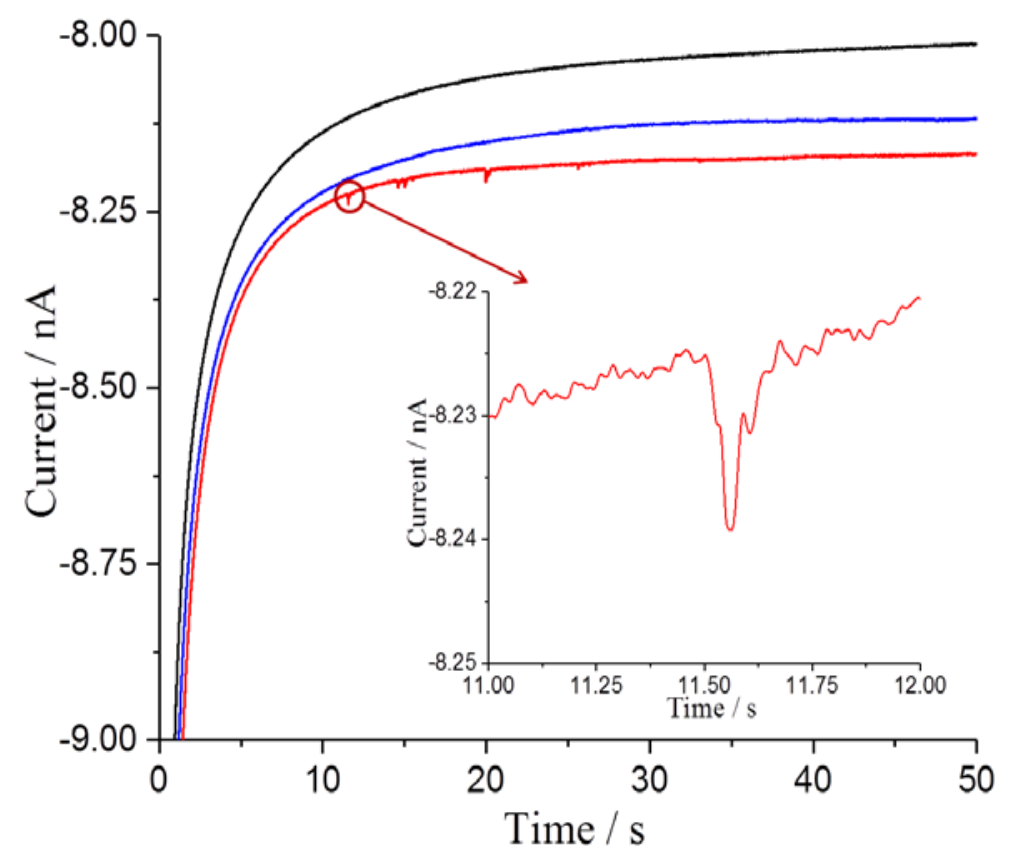

Figure 1: Representative current profiles during impact electrochemistry. Chronoamperometry recorded of $0.875 \mathrm{mM}$ TMPD-BF 4 in PBS solution at $\mathrm{pH} 7.4$ (black curve), and in the presence of $1.8 \times 10^{9} \mathrm{E}$. coli bacteria $\mathrm{mL}^{-1}$ (red curve) and in the presence of $1.8 \times 10^{9}$ bacteria $\mathrm{mL}^{-1}$ after 10 min exposure to $1 \%$ Virkon (blue curve), while applying a potential of $-0.15 \mathrm{~V} v \mathrm{~s}$ SCE.

As a proof of concept, various bacteria concentrations at a range of $3 \times 10^{8}$ bacteria $\mathrm{mL}^{-1}$ up to $1.8 \times 10^{9}$ bacteria $\mathrm{mL}^{-1}$ were analysed. The experiment at each concentration was repeated to obtain a large sample size. The number of impacts as function of time provides valuable information on the transport of the bacteria to the electrode ${ }^{17,26}$ hence providing a practical way to measure the concentration of the bacteria in an unknown sample. The theoretical value for the average impact frequency $\left(\mathrm{IF}_{\text {theory }} /\right.$ impacts s $\left.^{-1}\right)$ is established following the equation ${ }^{26,27}$

$$
I F_{\text {theory }}=4 D C r
$$

where $D$ is the diffusion coefficient of the target bacteria $\left(3.7 \times 10^{-9} \mathrm{~cm}^{2} \mathrm{~s}^{-1}\right), C$ is the E. coli concentration (CFU mL $\left.{ }^{-1}\right)$ and $r$ is the radius of the microelectrode $\left(2.2 \times 10^{-5}\right.$ 
m). The diffusion coefficient for living E. coli was obtained from literature where this parameter was determined using differential dynamic microscopy. ${ }^{28}$

We would like to point out that the use of either numbers of bacteria or the term "CFU" in this manuscript needs to be handled with care. In the previous sections, bacteria concentrations were stated as "number of bacteria" for solutions, as both living and dead bacteria had to be assumed. In contrast, when determining a concentration of bacteria from spikes during impact electrochemistry, as presented in the following, the use of CFU is appropriate, as only living organisms contribute to the observed “on”-signal. As shown in figure 2, the recorded experimental data is in close agreement with theory. The average impact frequency as a function of bacteria concentration follows a linear behaviour, which is in good agreement with the theory up to a concentration of about $9 \times 10^{8} \mathrm{CFU} \mathrm{mL}^{-1}$. In fact, the linear regression at concentrations of $3 \times 10^{8} \mathrm{CFU} \mathrm{mL} \mathrm{mL}^{-1}$ to $6 \times 10^{8} \mathrm{CFU} \mathrm{mL} \mathrm{mL}^{-1}$ bacteria follows a slope of $(2.91 \pm 0.028) \times 10^{-11} \mathrm{CFU} \mathrm{s}^{-1}$, which is in agreement with the theoretical slope of 3.30 $\times 10^{-11} \mathrm{CFU} \mathrm{s}^{-1}$. For low concentrations, chronoamperometry for a total duration of about 2000 seconds was recorded to ensure statistical validity of the measurements. Variations between expected and experimental currents are attributed to natural agglomeration of bacteria in solution. Previously, an influence on the current magnitude by shielding effects resulting from the insulating sheath of the microelectrode was reported ${ }^{29}$ and an effect on the current by any partial blocking of the electroactive surface. ${ }^{30}$ The deviation at higher bacteria concentration may be due to a destabilization of the bacteria single cell suspension, due to increased agglomeration, as well as a disturbance of the normal bacterial metabolism or even cell death as a result of a competition for oxygen in such highly dense suspensions. Although the reported detection of E. coli by impact chemistry is not selective yet, it is 
a valuable proof of concept, as it demonstrates a label free detection method for living pathogenic bacteria at clinically relevant concentrations. Thereby the recognition of $E$. coli is achieved within minutes. The relevance of the proposed method can be seen when noting that $E$. coli bacteria are one of the main pathogens in the food industry responsible for infections, where they exist in high numbers. This is due to their high proliferation rate, even at room temperature, which lets $E$. coli outgrow other bacteria rapidly. Therefore the concentrations tested in the presented research appear reasonable for point of care analysis. To achieve the recognition of lower E. coli concentrations in food sources, sample pre-concentrations can be conducted. Even under conditions of cross contaminations, we believe that the proposed method might become useful to provide a first alert for the presence of pathogens, which can then be further identified by more rigorous techniques.

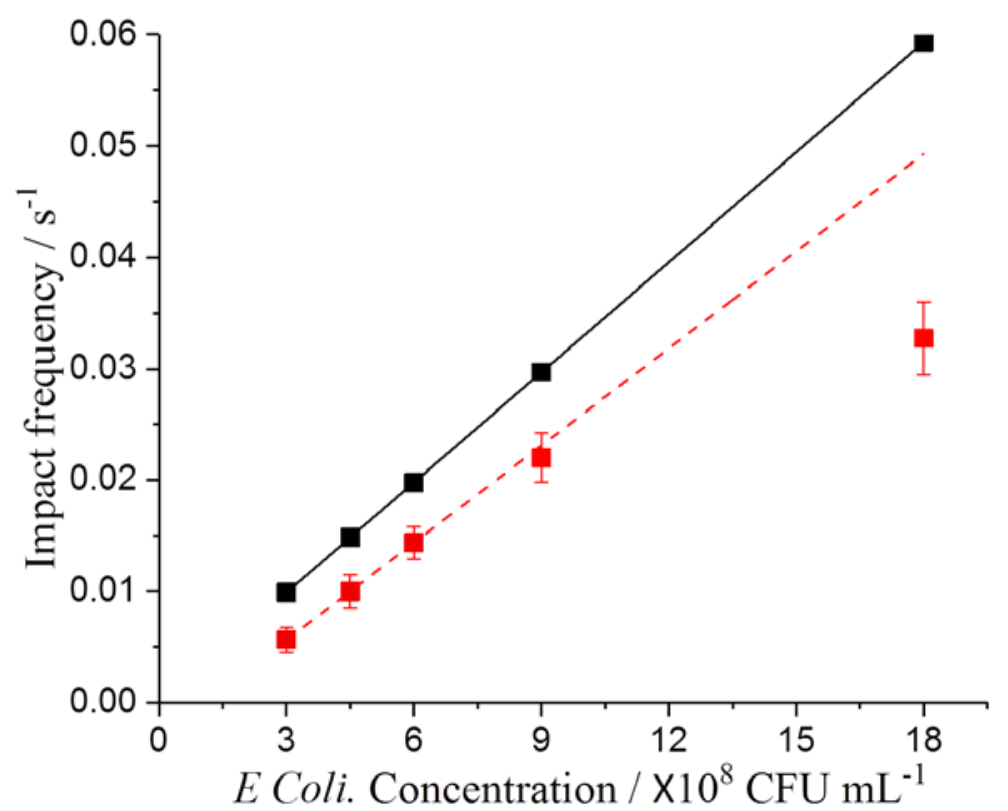

Figure 2: Theoretical (black) and experimental (red) average impact frequency as a function of varying E. coli concentrations. 
In conclusion, the electrochemical principle demonstrated in this study allows the label-free detection of $E$. coli bacteria in solution within seconds. The use of TMPD as redox mediator, which is interacting with bacterial cytochrome c oxidases, results in electrochemical current "on"-signals in the presence of E. coli, minimizing false positive signals due to non-electroactive impurities. The average impact frequency reported in this study scales linearly up to a bacteria concentration of $9 \times 10^{8} \mathrm{CFU} \mathrm{mL}^{-}$ ${ }^{1}$. The incorporation of this principle into microfluidic devices may allow the detection of even lower concentrations of bacteria and open the door for its application in the food industry or health services.

\section{Conflicts of interest}

The authors declare no competing financial interests.

\section{Acknowledgement}

The research leading to these results has received partial funding from the European Research Council under the European Union's Seventh Framework Program (FP/2007-2013)/ ERC Grant Agreement no. [320403]. RASC wishes to acknowledge the Portuguese Fundação para a Ciência e a Tecnologia (FCT) for her PhD fellowship (PD/BD/127797/2016) from PhD Programme in Medicines and Pharmaceutical Innovation (i3DU). 


\section{References}

1. C. L. Satterwhite, E. Torrone, E. Meites, E. F. Dunne, R. Mahajan, M. C. B. Ocfemia, J. Su, F. J. Xu and H. Weinstock, Sex Transm Dis, 2013, 40, 187-193.

2. Department of Health, Annual Report of the Chief Medical Officer: Infections and the rise of antimicrobial resistance, 2011.

3. D. Ivnitski, I. Abdel-Hamid, P. Atanasov, E. Wilkins and S. Stricker, Electroanal, 2000, 12, 317-325.

4. J. Monzo, I. Insua, F. Fernandez-Trillo and P. Rodriguez, Analyst, 2015, 140, 71167128.

5. C. Kokkinos, A. Economou and M. I. Prodromidis, Trac-Trend Anal Chem, 2016, 79, 88-105.

6. C. I. L. Justino, A. C. Duarte and T. A. P. Rocha-Santos, Trac-Trend Anal Chem, 2016, 85, 36-60.

7. United States Environmental Protection Agency, Recreational Water Quality Criteria, 2012.

8. $\quad$ Federal Register - Food and Drug Administration, Standards for the Growing, Harvesting, Packing, and Holding of Produce for Human Consumption, 2013.

9. Water UK, Water UK Briefing Note: E. coli (Escherichia coli) O157, 2006.

10. J. H. Kwon, M. K. Fausone, H. Y. Du, A. Robicsek and L. R. Peterson, Am J Clin Pathol, 2012, 137, 778-784.

11. A. Rompre, P. Servais, J. Baudart, M. R. de-Roubin and P. Laurent, J Microbiol Meth, 2002, 49, 31-54.

12. T. Albrecht, S. Horswell, L. K. Allerston, N. V. Rees and P. Rodriguez, Curr Opin Electroche, 2018, 7, 138-145.

13. K. J. Stevenson and K. Tschulik, Curr Opin Electroche, 2017, 6, 38-45.

14. Y. G. Zhou, N. V. Rees and R. G. Compton, Angew Chem Int Edit, 2011, 50, 42194221.

15. J. G. S. Moo and M. Pumera, ACS Sensors, 2016, 1, 949-957.

16. H. Wang and M. Pumera, Advanced Functional Materials, 2018, 28, 1705421.

17. L. Sepunaru, K. Tschulik, C. Batchelor-McAuley, R. Gavish and R. G. Compton, Biomaterials Science, 2015, 3, 816-820.

18. J. Y. Lee, B. K. Kim, M. Kang and J. H. Park, Sci Rep-Uk, 2016, 6.

19. S. Kuss, E. E. L. Tanner, M. Ordovas-Montanes and R. G. Compton, Chemical Science, 2017, 8, 7682-7688.

20. T. J. Cardwell, J. Mocak, J. H. Santos and A. M. Bond, Analyst, 1996, 121, 357-362.

21. C. Batchelor-McAuley, J. Ellison, K. Tschulik, P. L. Hurst, R. Boldt and R. G. Compton, Analyst, 2015, 140, 5048-5054.

22. Agilent Technologies, E.coli Cell Culture Concentration from OD600, http://www.genomics.agilent.com/biocalculators/calcODBacterial.jsp, (accessed 1 July 2018).

23. J. Yamauchi and H. Fujita, B Chem Soc Jpn, 1990, 63, 2928-2932.

24. E. Katelhon, E. E. L. Tanner, C. Batchelor-McAuley and R. G. Compton, Electrochimica Acta, 2016, 199, 297-304.

25. J. Winter, M. Ilbert, P. C. F. Graf, D. Ozcelik and U. Jakob, Cell, 2008, 135, 691-701.

26. S. V. Sokolov, S. Eloul, E. Kätelhön, C. Batchelor-McAuley and R. G. Compton, Physical Chemistry Chemical Physics, 2017, 19, 28-43.

27. D. Shoup and A. Szabo, Journal of Electroanalytical Chemistry, 1982, 140, 237-245. 
28. A. Jepson, V. A. Martinez, J. Schwarz-Linek, A. Morozov and W. C. K. Poon, Phys Rev E, 2013, 88.

29. S. Eloul and R. G. Compton, Chemelectrochem, 2014, 1, 917-924.

30. E. Katelhon, W. Cheng, C. Batchelor-McAuley, K. Tschulik and R. G. Compton, Chemelectrochem, 2014, 1, 1057-1062. 